\section{Outcome of surgery in patients with giant retinal tear: 10 years experience}

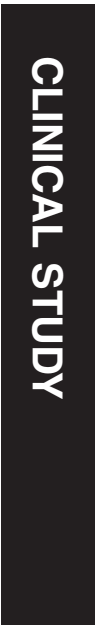

${ }^{1}$ Department of Ophthalmology, Eye Research Center, Rassoul Akram Hospital, Iran University of Medical Sciences, Tehran, Iran

${ }^{2}$ Student Research Committee, Faculty of Medicine, Iran University of Medical Sciences, Tehran, Iran

Correspondence: SA Alemzadeh, Department of Ophthalmology, Eye Research Center, Rassoul Akram Hospital, Iran University of Medical Sciences, SattarkhanNiayesh Street, Tehran 14456-13131, Iran Tel/Fax: +982166558811 . E-mail: s.amirpooya. alemzadeh@gmail.com

Received: 28 December 2016

Accepted in revised form: 22 June 2017

Published online:

4 August 2017

\author{
Abstract \\ Purpose To present our experience with \\ the surgery in retinal detachment (RD) \\ associated with giant retinal tear (GRT) \\ over $\mathbf{1 0}$ years in a tertiary referral \\ hospital. \\ Patients and Methods In this retrospective \\ study, the charts of all patients with the \\ diagnosis of RD associated with GRT who \\ underwent surgery from 2005 to 2015 at \\ Rassoul Akram Hospital were reviewed. \\ Exclusion criteria were concomitant presence \\ of diabetic retinopathy, and uveitis. All \\ patients had to have at least 3 months of \\ follow up. The success rate and factors \\ associated with repeated surgery were \\ determined. \\ Results Sixty two eyes of 61 patients \\ including $\mathbf{5 1}$ males and $\mathbf{1 0}$ females were \\ assessed. Proliferative vitreoretinopathy \\ (PVR) was present in $14(22.6 \%)$ of eyes. \\ Pars plana vitrectomy (PPV) alone was \\ performed in 44 eye $(71.0 \%)$ and \\ simultaneous vitrectomy and \\ phacoemulsification surgery was performed \\ in 18 eyes (29.0\%). An encircling episcleral \\ band was placed in 7 eyes (11.3\%). Anatomic \\ success after one vitrectomy procedure was \\ achieved in 45 eyes $(72.58 \%)$ and ultimately \\ in 61 eyes $(98.4 \%)$ at last follow up. \\ Seventeen eyes needed repeated PPV due to \\ redetachment associated with PVR in the \\ follow up period. The rate of repeated PPV \\ was significantly higher in eyes with PVR at \\ baseline and surgery with encircling \\ episcleral band. Trauma, extension of tear, \\ age, and lens status had no significant effect \\ on the rate of repeated PPV. \\ Conclusions Our study shows that the high \\ surgical success can be achieved in patients \\ with RD associated with GRT with single or \\ multiple surgeries. \\ Eye (2017) 31, 1284-1289; doi:10.1038/eye.2017.145; \\ published online 4 August 2017
}

K Ghasemi Falavarjani', SA Alemzadeh², M Modarres'1, SA Alemzadeh', MM Parvarash'1, M Naseripour ${ }^{1}, \mathrm{M} \mathrm{Hashemi}^{1}$ and M Robatmeili ${ }^{1}$

\section{Introduction}

Giant retinal tear (GRT) is defined as a fullthickness circumferential break in the neurosensory retina, extending more than 3 clock hours $\left(90^{\circ}\right) .{ }^{1,2}$ The majority of GRTs have been described to be idiopathic; however, GRT has been reported after trauma, extensive cryotherapy, photocoagulation and refractive surgery. 3,4

Historically, several surgical procedures including intentional retinal incarceration, prone air-fluid exchange and retinal tacks or sutures have been employes for the treatment of rhegmatogenous retinal detachment (RRD) associated with GRT. However, the outcomes were generally poor. ${ }^{5}$ Pars plana vitrectomy (PPV) is the standard treatment for RRD associated with GRT. ${ }^{6}$ Few case series have reported outcomes of surgery for RRD associated with GRT. ${ }^{1-5}$ These reports are generally small and the surgical techniques are different. Final success after single or multiple surgeries has been reported to be between 48 to $100 \%{ }^{2-7}$ Several factors including prior vitrectomy, GRT size greater than 180 and the presence of preoperative proliferative vitreoretinopathy (PVR) have reported to be associated with poor surgical success. ${ }^{7,8}$ Also, several innovations in the surgical instrumentations and techniques have improved the success rate of the surgery for GRTs. These include, but not limited to wideangle viewing systems, small gauge vitrectomy instruments, and introduction of perfluorcarbon liquids (PFCL). ${ }^{5}$

The aim of this study was to present our experience with the surgery in RRD associated with GRT over 10 years in a tertiary referral hospital.

\section{Methods}

In this retrospective non-comparative case series, the charts of all patients with the diagnosis of 
RRD associated with GRT who underwent surgery from 2005 to 2015 at Rassoul Akram Hospital were reviewed. Ethics committee of the Iran University of Medical Sciences approved the study. Exclusion criteria were concomitant presence of any other ocular pathology which could decrease visual acuity such as diabetic retinopathy, advanced glaucoma and previous uveitis at presentation. Data extracted were age, sex, duration of symptoms, extent of tear, types of surgery, lens status, PVR, status of the macula, involvement of fellow eye, number of additional surgery, anatomic outcome of first and final surgery and pre and postoperative visits' best corrected visual acuity (BCVA). Grading of PVR was performed based on the current accepted classification of PVR. ${ }^{9}$ Visual acuities were measured with Snellen chart and the measurements were converted to LogMAR for analysis. Refractive error was determined in eyes with attached macula. In others, the present glass reading or previous refraction was entered as refractive error. All patients had to have at least 3 months of follow up.

For all eyes, a standard 3 port pars plana vitrectomy was performed. The vitrectomy was performed with 20 gauge and 23 gauge instruments. The vitrectomy machine was Accurus (Alcon, Alcon Laboratories, TX) or Stellaris PC (Bausch and Lomb Incorporated, Bridgewater, NJ, USA). Combined phacoemulsification and PPV was done at surgeon discretion. After a core vitrectomy, posterior vitreous detachment was induced if needed and vitreous base shaving was performed. The retina was flattened using PFCL. The decision for placing a circumferential episcleral bands was made on discretion of the surgeon. Silicone oil (1000, 5000, or heavy silicone oil, Bausch and Lomb, Toulouse, France) was injected for retinal tamponade. Subconjunctival antibiotics and steroids were injected at the conclusion of the surgery.

Data analysis was performed using SPSS software (version 15, SPSS Inc., Chicago, IL, USA). Student's $t$-test, paired $t$-test, Wilcoxon Test, $X^{2}$-test, Fisher's exact Test, Friedman Test and Multivariate regression analysis were used for analysis. $P$-value $<0.05$ was considered significant. The 10-year period of the study, was divided into the two 5-year periods. The surgical success rates were compared between the two periods of time.

\section{Results}

Ninety five patients with the diagnosis of RRD associated with GRT during 10 years were reviewed. Thirty three patients were excluded due to inadequate follow up. Finally, 62 eyes of 61 patients with GRT were studied. Ten patients $(16.4 \%)$ were female and 51 patients $(83.6 \%)$ were male. Demographics and baseline characteristics are summarized in Table 1. No eye had a diagnosis of
Table 1 Demographics and baseline characteristics

\begin{tabular}{|c|c|}
\hline Baseline characteristics & $\begin{array}{l}\text { Number of patients: } 61 \\
\text { Number of eyes: } 62\end{array}$ \\
\hline Mean age $\pm S D$, (range) years & $33.96 \pm 20.19(4-76)$ \\
\hline \multicolumn{2}{|l|}{$\operatorname{Sex}, n(\%)$} \\
\hline Male & $51(83.6)$ \\
\hline Female & $10(16.4)$ \\
\hline Mean follow-up $\pm S D$,(range) months & $21.54 \pm 27.73(3-126)$ \\
\hline Mean Refraction $\pm S D,($ range $)$ & $-4.78 \pm 8.98(-24-16)$ \\
\hline \multicolumn{2}{|l|}{ Years of surgery } \\
\hline First 5 years period & $22(35.5)$ \\
\hline Second 5 years period & $40(64.5)$ \\
\hline \multicolumn{2}{|l|}{ Lens status, $n(\%)$} \\
\hline Phakic/no cataract & $33(53.2)$ \\
\hline Phakic/cataract & 9 (14.5) \\
\hline Pseudophakic & $14(22.6)$ \\
\hline Aphakic & $6(9.7)$ \\
\hline \multicolumn{2}{|l|}{ Eye, $n(\%)$} \\
\hline OD & $30(48.4)$ \\
\hline OS & $32(51.6)$ \\
\hline History of trauma $n(\%)$ & $18(29.03)$ \\
\hline Proliferative vitreoretinopathy, $n(\%)$ & $14(22.6)$ \\
\hline $\begin{array}{l}\text { Preoperative number of eyes with } \\
\text { anterior PVR }\end{array}$ & $10(16.1)$ \\
\hline $\begin{array}{l}\text { Preoperative number of eyes with } \\
\text { posterior PVR }\end{array}$ & $11(18.0)$ \\
\hline $\begin{array}{l}\text { Preoperative extent of anterior PVR } \\
\text { (clock hour) }\end{array}$ & $5.42 \pm 1.98$ \\
\hline $\begin{array}{l}\text { Preoperative extent of posterior PVR } \\
\text { (clock hour) }\end{array}$ & $7.00 \pm 4.76$ \\
\hline Macula detachment, $n(\%)$ & $51(82.3)$ \\
\hline \multicolumn{2}{|l|}{ Extent of GRT } \\
\hline$<180$ & $42(67.8)$ \\
\hline$>180$ & $20(32.2)$ \\
\hline
\end{tabular}

hereditary vitreoretinopathy. Thirteen eyes (21\%) were high myopic.

Fifty one eyes (82.3\%) had a detached macula. The GRT was $180^{\circ}$ or greater in $20(32.2 \%)$ eyes and less than $180^{\circ}$ in $42(67.8 \%)$ eyes. Proliferative vitreoretinopathy was present in 14 (22.6\%) eyes. PVR was grade C in all 14 eyes. PVR was anterior in 10 eyes and posterior in 11 eyes. The extensions was from 4 to 12 o'clock in anterior and from 2 to 12 o'clock in posterior PVR.

PPV alone was performed in 44 eye $(71.0 \%)$ and simultaneous PPV and phacoemulsification surgery was performed in 18 eyes (29.0\%). Phacoemulsification was performed for significant cataract in 10 eyes, and subluxation of the lens in one eye. Seven patients had clear lens; these patients underwent combined surgery to provide better visualization of peripheral retina. An encircling scleral band (No. 240, FCI Inc., Paris, France) was placed in 7 eyes $(11.3 \%)$. The reason for placing the episcleral band was the age ( $\leq 18$ years) in 4 eyes. In 3 
eyes, the encircling band was placed for prevention of RD in case of future PVR. All eyes received silicone oil tamponade and had 360 degree laser photocoagulation of the peripheral retina, intraoperatively. Heavy silicone oil (Oxane HD; Bausch and Lomb, Toulouse, France), was injected in 10 (16.1\%) eyes.

Patients were followed for a mean of $21.54 \pm 27.73$ 126) months. All eyes had 3 months of follow up. Six, 12 and 24 month follow up was available for 52, 32 and 18 eyes. Anatomic success after one PPV procedure was achieved in 45 eyes (72.58\%). Final anatomical surgical success was achieved in 61 eyes (98.4\%) at last follow up. The single case of anatomical failure had localized inferior retinal detachment 4 months after primary surgery and refused additional surgery. Seventeen eyes needed repeated PPV due to recurrent RRD associated with PVR. For 8 eyes, the primary surgery date was in the first 5 -year

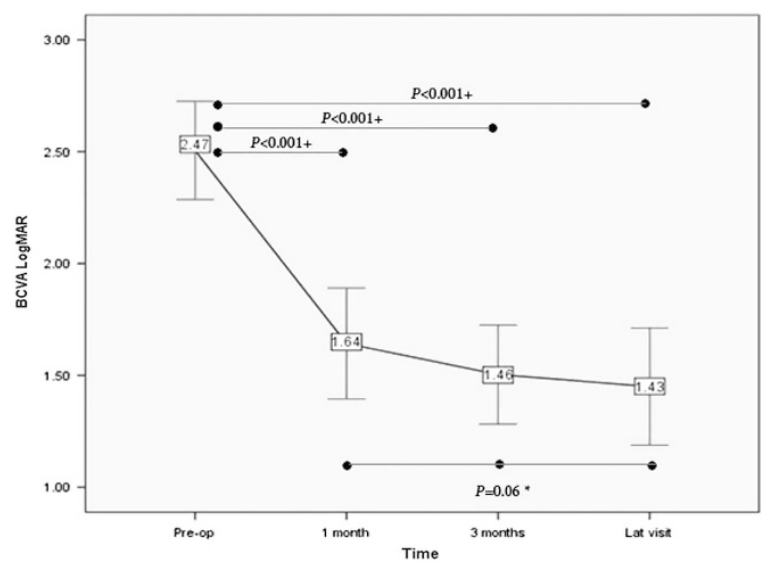

Figure 1 Preoperative and postoperative best corrected visual acuity. ${ }^{*}$ Friedman Test, +Wilcoxon Test. and for 9 eyes was in the second 5-year study period $(P=0.24)$. Fourteen eyes needed one, two eyes needed 2 and one eye needed 3 additional PPV surgeries. At the end of follow up, silicone oil had been removed from 41 eyes $(66.1 \%)$.

Mean preoperative BCVA was $2.47 \pm 0.79$ LogMAR at baseline, and $1.64 \pm 0.85$ and $1.46 \pm 0.75 \operatorname{LogMAR}$ at 1 and 3 months after surgery, respectively (both $P<0.001$,

Figure 1). At the last visit, mean BCVA was $1.43 \pm 0.92$ LogMAR $(P<0.001)$. BCVA improved one line or more in $70 \%$ and 3 lines or more in $68 \%$ of the patients, respectively.

The rate of repeated PPV was significantly correlated with baseline PVR and placement of encircling episcleral band $(\mathrm{OR}=3.8, P=0.03$ and $\mathrm{OR}=24.00, P=0.005$, respectively). Trauma, high myopia, time period of surgery, extension of tear, age, and lens status had no significant effect on the rate of repeated PPV (Table 2).

In multivariate logistic regression analysis both PVR and encircling band remained significantly associate with repeated $\mathrm{PPV}(\mathrm{OR}=5.39 P=0.01$ and $\mathrm{OR}=38.58$, $P=0.002$ respectively).

\section{Discussion}

In this study, we found a high anatomical reattachment rate of $72.5 \%$ after first surgery with a final success rate of $98.4 \%$. The age range and the sex in our study population was similar to those reported in previous studies. ${ }^{7-11} \mathrm{~A}$ history of blunt trauma was found in $29 \%$ of eyes. Previous studies have also reported a similar rate of trauma (10-40\%). . $^{3,4}$ Similarly, the rate of high myopia in this study $(21 \%)$ was within the range of previous reports (12-47\%). ${ }^{7-11}$ In those studies that the presence of PVR was not among the exclusion criteria, the rate of PVR was

Table 2 Patients characteristics in single surgery versus repeated PPV group

\begin{tabular}{|c|c|c|c|}
\hline & Single surgery & Repeated PPV & $\mathrm{P}$ value \\
\hline Number & 45 & 17 & \\
\hline Age & $35.6 \pm 20.02$ & $29.41 \pm 20.54$ & $0.27^{\mathrm{a}}$ \\
\hline $\operatorname{Sex}(\mathrm{M} / \mathrm{F})$ & $36 / 9$ & $16 / 1$ & $0.17^{\mathrm{b}}$ \\
\hline Age group (under $20 /$ more than 20 ) & $14 / 31$ & $9 / 8$ & $0.16^{\mathrm{c}}$ \\
\hline Eye $(\mathrm{OD} / \mathrm{OS})$ & $20 / 25$ & $10 / 7$ & $0.31^{\mathrm{c}}$ \\
\hline PVR at presentation & 7 & 7 & $0.03^{\mathrm{b}}$ \\
\hline Lens status (Phakic/Pseudophakic/Aphakic) & $31 / 11 / 3$ & $11 / 3 / 3$ & $0.59^{c}$ \\
\hline Tear extension $(<180 />180)$ & $32 / 13$ & $10 / 7$ & $0.23^{c}$ \\
\hline Trauma & 11 & 7 & $0.19^{c}$ \\
\hline Time period of surgery (first 5 years period/second five period) & $14 / 31$ & $8 / 9$ & $0.24^{\mathrm{c}}$ \\
\hline High myopia & 8 & 5 & $0.50^{\mathrm{b}}$ \\
\hline SO removal & 32 & 9 & $0.17^{\mathrm{c}}$ \\
\hline Phacovitx/Vitx alone & $12 / 33$ & $6 / 11$ & $0.50^{c}$ \\
\hline Surgery type (without band/with band) & $44 / 1$ & $11 / 6$ & $0.00^{\mathrm{b}}$ \\
\hline
\end{tabular}

${ }^{\text {a }}$-test. ${ }^{b}$ Fisher's exact test. $\chi^{c}$. 


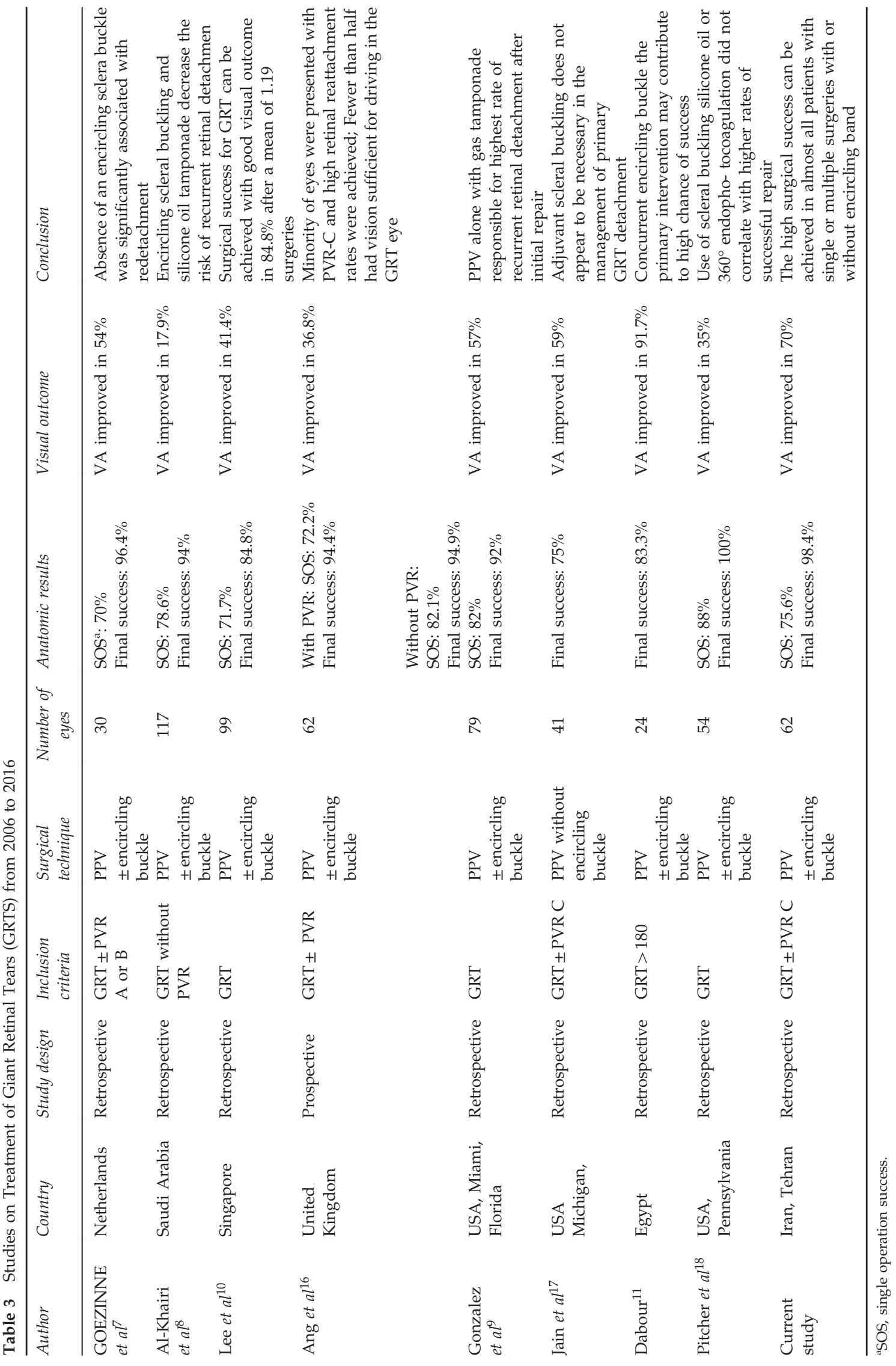


between $9 \%$ to $41 \%$. 2,10 The rate of baseline PVR was $22.6 \%$ in our series.

Recent advances in the surgical techniques and instruments improved the success of the surgery for RRD associated with GRT. ${ }^{12}$ Primary and final retinal reattachment rates after repeated PPV vary widely among published studies, which may be partly related to the wide variability in preoperative characteristics of the GRT associated RRD as well as the surgical techniques. The single operation success ranged from 70 to $88 \%$. We achieved a single operation success rate of $72.58 \%$ and final anatomic success rate of $98.4 \%$ at last follow up. Table 3 summarizes the recent large studies reported the results of PPV for the treatment of RRD associated with GRT. During the recent 10 years, 3 studies reported a higher number of eyes compared to our study. The largest recent series is a retrospective study of 117 eyes with GRT without PVR, reported a primary procedure's success rate of $78.6 \%$ which increased to $94 \%$ with multiple surgeries. ${ }^{7}$ They used an encircling scleral buckle in 97 $(82.9 \%)$, silicone oil tamponade in $64(54.7 \%)$ and perfluoropropane gas in $53(45.3 \%)$ eyes. Finally the study concluded higher success rate in primary surgery was achieved with placement of encircling band and use of silicone oil tamponde. Lee and colleagues ${ }^{10}$ reported 99 eyes of 96 patients with a single operation success of 71.8 that increased to $84.8 \%$ finally with a mean of 1.19 surgeries. In their study, the prevalence of high myopia (40.6\%) was higher and in 90 eyes $(70.3 \%)$, encircling scleral buckling was placed. In another study by Gonzalez and associates, 1279 eyes of 77 patients were reported. Primary success was $82 \%$ with a single surgery and $92 \%$ at final visit. They reported that history of trauma, PVR and gas tamponade were associated with higher rates of recurrent retinal detachments. We could not find any association between baseline demographics and the rate of retinal redetachment except for PVR. Similarly, Scott ${ }^{3}$ and Glaser ${ }^{13}$ found that the baseline PVR is a risk factor for future redetachment.

A wide variation in final visual outcomes has been reported in previous studies. The final BCVA has been reported to be $20 / 40$ or better in $0-50 \%$ and $20 / 200$ or lower in $10-58 \%$ of patients in different studies. ${ }^{4,14-17}$ In our study, the baseline BCVA was greater than 20/40 in $1.6 \%$ and lower than $20 / 200$ in the majority of eye (90\%), that is markedly lower than other studies, however, at final visit the BCVA significantly improved and $16 \%$ of eyes had BCVA greater than 20/40.

Use of a supplemental encircling band in managing GRTs remains controversial. Some studies reported higher anatomical success and low redetachment rates in PPV surgeries combined with encircling buckle ${ }^{6,10}$ while others showed high success rate with vitrectomy alone. ${ }^{18}$ In the current study, placement of encircling band was significantly associated with repeated PPV( OR $=38.58$ in multivariate logistic regression model). Considering the retrospective nature of this study this maybe due to the fact that surgeon had placed encircling band in eyes in which they predicated worse prognosis such as beginning of PVR or stiffness of retina. Alternatively, some have reported that scleral buckle placement in the eyes with GRT may intervene with retinal reattachment. ${ }^{19,20}$ Also, we placed a $2.5 \mathrm{~mm}$ wide episcleral band (No. 240) to support the vitreous base. Placing a wider scleral buckle may result in different outcomes. Removal of the lens is often performed to enable better visualization of the peripheral retina. In our study lens was removed in $29 \%$ eyes, whereas this rate was $41 \%$ to 51.2 in other studies. ${ }^{12,15}$ This difference may be due to lower percentage of eyes with PVR in our study.

Present study is limited by its retrospective nature, lack of control group and the small sample size. Also, nearly one third of the patients were excluded due to the inadequate follow up. This may cause a bias into the study results. Silicone oil was removed from 41 eyes during follow up. Although we defined the success rate according to the absence of RD associated with PVR, the retina could be detached due to missed retinal breaks after silicone removal. Despite these limitations this is one of the largest series reported from the Middle East. Our study shows that the high surgical success can be achieved in almost all patients with single or multiple surgeries. Patients should be aware the repeated surgeries may be need to save the eye. Future studies are needed to assess the role of episcleral encircling band on the surgical outcomes.

\section{Summary}

What was known before

- Management of GRTs is complicated. a.Final success after single or multiple surgeries has been reported to be between 48 to $100 \%$.

- Using encircling band is controversial. a.Some studies reported higher anatomical success and low redetachment rates in PPV surgeries combined with encircling buckle6, 13 while others showed high success rate with vitrectomy alone.

What this study adds

- Current study is one of the largest series reported from the Middle East.

- In the present study single operation success rate was $72.58 \%$ and final anatomic success rate was $98.4 \%$ at last follow up.

- Current study shows that the high surgical success can be achieved in almost all patients with single or multiple surgeries. 


\section{Conflict of interest}

The authors declare no conflict of interest.

\section{References}

1 Mathis A, Pagot V, Gazagne C, Malecaze F. Giant retinal tears: surgical techniques and results using perfluorodecalin and silicone oil tamponade. Retina 1992; 12: S7-S10.

2 Kertes PJ, Wafapoor H, Peyman GA, Calixto Jr N, Thompson $\mathrm{H}$. The management of giant retinal tears using perfluoroperhydrophenanthrene: a multicentre case series. Vitreon Collaborative Study Group. Ophthalmology 1997; 104: 1159-1165.

3 Scott IU, Murray TG, Flynn HW, Feuer WJ, Schiffman JCPerfluoron Study Group. Outcomes and complications associated with giant retinal tear management using perfluoro-n-octane. Ophthalmology 2002; 109(10): 1828-1833.

4 Ambresin A, Wolfensberger TJ, Bovey EH. Management of giant retinal tears with vitrectomy, internal tamponade, and peripheral 360 degrees retinal photocoagulation. Retina 2003; 23: $622 \mathrm{e} 8$.

5 Berrocal MH, Chenworth ML, Acaba LA. Management of giant retinal tear detachments. J Ophthalmic Vis Res 2017; 12(1): 93-97.

6 Ghosh YK, Banerjee S, Savant V, Kotamarthi V, Benson MT, Scott RA et al. Surgical treatment and outcome of patients with giant retinal tears. Eye 2004; 18: 996e1000.

7 Goezinne F, LAH EC, Berendschot TT, Gast ST, Liem AT, Lundqvist IL et al. Low redetachment rate due to encircling scleral buckle in giant retinal tears treated with vitrectomy and silicone oil. Retina 2008; 28(3): 485-492.

8 Al-Khairi AM, Al-Kahtani E, Kangave D, Abu El-Asrar AM. Prognostic factors associated with outcomes after giant retinal tear management using perfluorocarbon liquids. Eur J Ophthalmol. 2008; 18: $270 \mathrm{e}$.

9 Machemer R, Aaberg TM, Freeman HM, Irvine AR, Lean JS, Michels RM. An updated classification of retinaldetachment with proliferative vitreoretinopathy. Am I Ophthalmol 1991; 112: 159-165.

10 Lee SY, Ong SG, Wong DWK, Ang CL. Giant retinal tear management: an Asian experience. Eye 2009; 23(3): 601-605.

11 Dabour SA. The outcome of surgical management for giant retinal tear more than 180'. BMC Ophthalmol 201427 14: 86.

12 Gonzalez MA, Flynn HW, Smiddy WE, Albini TA, Tenzel P. Surgery for retinal detachment in patients with giant retinal tear: etiologies, management strategies, and outcomes. Ophthalmic Surg Lasers Imaging Retina 2013; 44(3): 232-237.

13 Glaser BM. Treatment of giant retinal tears combined with proliferative vitreoretinopathy. Ophthalmology 1986; 93(9): 1193-1197.

14 Unlu N, Kocaoglan H, Acar MA, Sargin M, Aslan BS, Duman $S$. The management of giant retinal tears with silicone oil. Eur J Ophthalmol 2003; 13: 192-195.

15 Verstraeten T, Williams GA, Chang S, Cox Jr MS, Trese MT, Moussa $\mathrm{M}$ et al. Lens-sparing vitrectomy with perfluorocarbon liquid for the primary treatment of giant retinal tears. Ophthalmology 1995; 102: 17e20.

16 Ang GS, Townend J, Lois N. Epidemiology of giant retinal tears in the United Kingdom: the British Giant Retinal Tear Epidemiology Eye Study (BGEES). Invest Ophthalmol Vis Sci 2010; 51(9): 4781-4787.

17 Jain N, Kozak JA, Niziol LM, Musch DC, Zacks DN. Vitrectomy alone in the management of giant retinal tears. Ophthalmic Surg Lasers Imaging Retina 2014; 45(5): 421-427.

18 Pitcher JD 3rd, Khan MA, Storey PP, Hsiao-Fang-Yen N, Dollin ML, Hsu J et al. Contemporary management of rhegmatogenous retinal detachment due to giant retinal tears: a consecutive case series. Ophthalmic Surg Lasers Imaging Retina 2015; 46(5): 566-570.

19 Hoffman ME, Sorr EM. Management of giant retinal tears without scleral buckling. Retina 1986; 6(4): 197-204.

20 Kreiger AE, Lewis H. Management of giant retinal tears without scleral buckling. Use of radical dissection of the vitreous base and perfluoro-octane and intraocular tamponade. Ophthalmology 1992; 99(4): 491-497. 